\title{
CONHECIMENTOS DAS GRÁVIDAS EM SAÚDE MENTAL POSITIVA
}

\author{
Paula Nelas \\ Escola Superior de Saúde, Instituto Politécnico de Viseu, UICISA: E \\ pnelas@gmail.com \\ Cláudia Chaves \\ Escola Superior de Saúde, Instituto Politécnico de Viseu, Ci\&DEI \\ claudiachaves21@gmail.com \\ Emília Coutinho \\ Escola Superior de Saúde, Instituto Politécnico de Viseu, UICISA: E \\ ecoutinhoessv@gmail.com \\ João Duarte \\ Escola Superior de Saúde, Instituto Politécnico de Viseu, UICISA: E \\ duarte.johnny@gmail.com
}

Recepción Artículo: 17 mayo 2021 Admisión Evaluación: 17 mayo 2021 Informe Evaluador 1: 18 mayo 2021 Informe Evaluador 2: 21 mayo 2021 Aprobación Publicación: 01 junio 2021

\section{RESUMO}

A gravidez constitui um período de extrema importância enquanto momento de transição para a parentalidade. Decorrente das alterações biopsicossociais desta fase, torna-se pertinente analisar os fatores que promovem a literacia em saúde mental da grávida. São objetivos avaliar o grau de literacia em saúde mental positiva e analisar as variáveis promotoras da literacia em saúde mental, em grávidas. Trata-se de um estudo quantitativo, transversal, descritivo e correlacional, constituído por uma amostra de 222 grávidas, média de idade 30.45 anos (dp \pm 4.64$)$. 0 instrumento de colheita de dados foi um questionário, que permitiu caracterizar a amostra nas dimensões sociodemográfica e clínica. Foi ainda utilizada a escala de avaliação da literacia em saúde mental positiva de Chaves, Sequeira \& Duarte (2018). Em termos de resultados constatou-se que as grávidas com companheiro, residentes em zona urbana, com ensino superior, ativas profissionalmente, com uma profissão intelectual, sem hábitos tabágicos, que classificam a sua alimentação como saudável/muito saudável, revelam mais literacia em saúde mental positiva. Verificou-se ainda que as grávidas cuja gravidez foi planeada, que recorreram a tratamentos de fertilidade, que a gravidez é vigiada por enfermeiro e médico, que cumprem a vigilância da gravidez, que frequentam curso de preparação para o parto e parentalidade, apresentam mais literacia em saúde mental positiva. Recomendam-se programas de intervenção, como por exemplo, a realização de rastreio de saúde mental na grávida.

Palavras chave: grávida; saúde mental positiva; conhecimentos 


\section{CONHECIMENTOS DAS GRÁVIDAS EM SAÚDE MENTAL POSITIVA}

\section{ABSTRACT}

Pregnant women's knowledge of positive mental health. Pregnancy is an extremely important period as a moment of transition to parenting. Due to the biopsychosocial changes in this phase, it is pertinent to analyze the factors that promote mental health literacy of pregnant women. The objectives are to evaluate the level of positive mental health literacy and identify wich variables promote mental health literacy in pregnant women. This is a quantitative, cross-sectional, descriptive and correlational study, with a sample of 222 pregnant women, mean age 30.45 years $(S D \pm 4.64)$. The data collection instrument was a questionnaire, which allowed the characterization of the sample in the sociodemographic and clinical dimensions. The positive mental health literacy scale of Chaves, Sequeira \& Duarte (2018) was also used. In terms of results, it was found that pregnant women with a partner, living in an urban area, with higher education, professionally active, with an intellectual profession, without smoking habits, which classify their diet as healthy / very healthy, reveal more positive mental health literacy. It was also found that pregnant women whose pregnancy was planned, who resorted to fertility treatments, that the pregnancy is supervised by a nurse and doctor, who carry out pregnancy surveillance, who attend a preparation course for childbirth and parenting, have more literacy in positive mental health. Intervention programs are recommended, such as carrying out mental health screening on pregnant women, in each trimester of pregnancy, to promote mental health literacy.

Keywords: pregnant; positive mental health; knowledge

\section{INTRODUÇÃO}

Cada mulher vive a gravidez como uma experiência única que envolve modificações psíquicas, físicas, fisiológicas, sociais e emocionais. (Guerra, Braga, Quelhas \& Silva, 2014). A par da adaptação física, o ajuste psicológico é um fator determinante para o sucesso de transição para a parentalidade, o que implica atenção ao estado emocional e mental da grávida (Brito, 2009). Pelo que há necessidade de incrementar o conhecimento sobre a problemática da saúde mental neste período da vida da mulher, quer na compreensão dos fenómenos que a afetam, quer nas estratégias que promovem a sua saúde mental (Guerra, et al, 2014). Assim, a literacia em saúde mental é um desafio atual, permite melhorar os resultados da saúde da grávida, bem como da população em geral, possibilitando obter conhecimento e crenças sobre transtornos mentais que ajudam no seu reconhecimento, gestão ou prevenção (Jorm, 2012).

As transições são períodos complexos e multidimensionais, contudo existem algumas propriedades comuns identificadas, nomeadamente a consciência, o compromisso, a mudança e diferença, o período de experiência e os pontos críticos e eventos. 0 enfermeiro é um elemento facilitador da transição, como é 0 caso da transição para a parentalidade, pois é o profissional de saúde que mais lida com as condições que rodeiam 0 individuo e que podem facilitar ou inibir a experiência da transição. São os enfermeiros que têm conhecimento sobre as condições económicas, sociais e familiares que interferem com o processo de transição, e por isso, é que a transição é um foco importante nos cuidados de enfermagem (Meleis, Sawyer, Messias \& Schumacher, 2010).

As alterações no decurso da gravidez variam de mulher para mulher, consoante a sua personalidade, a sua história, o meio humano em que se envolveu, as relações estabelecidas com os seus próprios pais ou com o pai do seu filho. A expressão das experiências de cada mulher varia consoante a sua cultura, as suas ambições, os seus projetos, o seu funcionamento afetivo emocional. Porém, a integração equilibrada de todos estes aspetos psicológicos e culturais nem sempre é fácil, na medida em que muitas mulheres vivem conflitos psicológicos, requerendo um ajustamento psicológico.

As atitudes da grávida estão frequentemente associadas a alguma angústia e incerteza em relação à desejabilidade da gravidez. Assim, podem manifestar reações psicológicas aquando da confirmação da gravidez como por exemplo alterações de humor, oscilações dos sentimentos em relação ao marido e outras pessoas, podendo surgir depressão. No entanto, quando existe um projeto adaptativo de maternidade, a gravidez permite a preparação para ser mãe - ensaiar cognitivamente papéis e tarefas maternas, ligar-se efetivamente à criança, iniciar 0 
processo de reestruturação de relações para incluir o novo elemento, incorporar a existência do filho na sua identidade e, simultaneamente, aprender a aceitá-lo como pessoa única, com vida própria. Assim, o período gravídico é caracterizado por ensaios, ligação, ansiedade, fantasias e reflexões, que permitem que o projeto da maternidade se continue a construir e consolidar de forma progressiva (Alderdice, MacNeill \& Lynn, 2013).

Segundo Brito (2009), na gravidez, o bem-estar psicológico é importante, o que implica atenção ao estado emocional da grávida, para tal devem ser mobilizados os conviventes significativos (cônjuge/companheiro e de outros familiares próximos da mulher grávida). A mesma autora refere que são vários os fatores de risco que podem potenciar estados de perturbação psicológica na gravida, nomeadamente gravidez não planeada, não desejada e não aceite, não realização de preparação para o parto e parentalidade, gravidez de alto risco, malformações do feto, morte de um familiar próximo (mãe, cônjuge, pessoa significativa) durante a gravidez, patologia psiquiátrica, toxicodependências, aparecimento de situações de risco associadas a antecedentes obstétricos, interrupção in/ou voluntárias da gravidez, morte in útero, parto prematuro e gravidez anterior não vigiada. São também referenciados os fatores de risco relacionados com o contexto familiar e social do casal, tais como violência doméstica, que pode estar na base da depressão da mulher, isolamento social da grávida, ser grávida adolescente, défice nas relações com a família de origem, colocação familiar ou institucional na infância. Pelo exposto, a gravidez é tida como um período de vulnerabilidade para o desenvolvimento de transtornos psiquiátricos, pois estão presentes importantes alterações físicas, hormonais, psíquicas e de inserção social, que podem influenciar diretamente a sua Saúde Mental.

Em suma, a gravidez é um momento de transição grandemente significativo durante a vida da mulher, requerendo várias adaptações. A capacidade da mulher se adaptar às modificações e exigências da gravidez afeta a sua saúde física e mental, e parece ter igualmente influência na saúde do feto durante o período gestacional. Contudo, quando esta dispõe de um sistema de apoio, consegue enfrentar as mudanças e transições de desenvolvimento, diminuindo o impacto dos eventos adversos na sua saúde mental, levando-a a um maior ajustamento psicológico, particularmente quando os níveis de vulnerabilidade são elevados.

Para Loureiro (2015), a literacia em saúde é um importante determinante da saúde e da qualidade de vida, refletindo as desigualdades sociais. Incorpora fatores psicológicos, sociais e ambientais que influenciam as escolhas e os comportamentos relacionados com a saúde. Deste modo, a literacia em saúde é resultado de ações de promoção da saúde, de ambientes favorecedores de escolhas saudáveis, de educação para a saúde e de estratégias de empoderamento da população. Assim, é imprescindível o envolvimento de todos os setores da saúde, de forma a melhorar as competências dos cidadãos para lidarem com a própria saúde, melhorando a sua qualidade de vida. Para a autora, o estigma da doença mental e a relutância em relação a tudo 0 que envolve o psicológico são as principais causas da falta de procura de informação relativa à saúde mental. No entanto, sendo a gravidez um período que envolve significativas adaptações fisiológicas e psicológicas, torna-se necessário a existência de literacia em saúde mental, ou seja, a aquisição de competências e saberes, de forma a lidar mais harmoniosamente com as preocupações e ansiedades associados às tarefas do exercício da maternidade, capacitando-as a gerir de forma eficaz as condições de saúde ao longo do percurso (Kutcher, Wei \& Coniglio, 2016).

Para potenciar a literacia, são postas em prática, pelos profissionais de saúde, várias intervenções. As campanhas comunitárias têm a grande vantagem de facultar a promoção da literacia de forma global, não apenas a uma pessoa, mas a toda a comunidade em geral. Da mesma forma, sessões de educação para a saúde têm trazido vários contributos para a ampliação da literacia em saúde mental. A internet, como fonte de acesso fácil, tem sido o maior e mais utlizado recurso, para esclarecimento de dúvidas e ampliação de conhecimentos (Jorm, 2014). No entanto, a importância das consultas nos cuidados de saúde primários e diferenciados, como o elemento base para o funcionamento da saúde em Portugal, não deve ser substituída pelas tecnologias de comunicação e informação.

A intervenção por parte do profissional de enfermagem também é efetiva, na medida, que os enfermeiros devem prestar cuidados individualizados e flexíveis, estando consciencializados das circunstâncias sociais da 


\section{CONHECIMENTOS DAS GRÁVIDAS EM SAÚDE MENTAL POSITIVA}

grávida, especialmente, de situações onde sejam evidenciadas dificuldades de ajustamento. É um facto que também as grávidas devem esclarecer as suas dúvidas no âmbito da transição para a parentalidade, incrementando a sua literacia (Guerra et al, 2014). Deste modo, é necessário fornecer as bases para aumentar a literacia em saúde mental materna, assim como avaliá-la durante os cuidados prestados durante este período. Ao identificar os tópicos onde a grávida tem dificuldades em gerir as situações do dia-a-dia e onde tem conhecimento insuficiente (Kohan, Ghasem, \& Dodangeh, 2007). Neste sentido, é necessário ensinar às grávidas estratégias de gestão do stress/ansiedade, competências que possibilitarão uma vivência mais harmoniosa da maternidade. Estratégias de autoajuda são ações que a grávida pode adquirir e colocar em prática, de forma a gerir melhor as suas emoções, mantendo assim o seu bem-estar psicológico. De entre várias ações de autoajuda, Jorm (2012) ressalta a procura de apoio junto da família e amigos, o aumentar a atividade física e a prática de atividades de lazer.

\section{OBJETIVOS DA INVESTIGAÇÃO}

Avaliar o grau de literacia em saúde mental positiva em grávidas e analisar as variáveis promotoras da literacia em saúde mental, em grávidas.

\section{AMOSTRA E PARTICIPANTES}

Trata-se de uma amostra não probabilística por conveniencia, constituida por 222 grávidas com uma média de idade de 30.45 anos $(\mathrm{dp} \pm 4.64)$.

\section{METODOLOGIA E INSTRUMENTOS UTILIZADOS}

Trata-se de um estudo quantitativo, transversal, descritivo e correlacional. 0 instrumento de colheita de dados foi um questionário, que permitiu caracterizar a amostra nas dimensões sociodemográfica e clínica. Foi ainda utilizada a escala de avaliação da literacia em saúde mental positiva de Chaves, Sequeira \& Duarte (2018). 0 estudo foi submetido a registo e pedido de parecer da Comissão Nacional de Proteção de Dados. Foram respeitados os procedimentos éticos e legais relativos à aplicação e análise dos dados. 0 instrumento de cálculo foi 0 Statistical Package for the Social Sciences (SPSS), na versão 24.0 para Windows.

\section{RESULTADOS ALCANÇADOS}

A amostra é constituída por 222 mulheres que se encontram em período gravídico. Relativamente à idade, a percentagem mais elevada (43.2\%) corresponde às participantes com $\leq 29$ anos, seguindo-se as que possuem idade igual ou superior aos 33 anos (33.3\%) e 23.4\% situa-se na faixa etária dos 30-32 anos. A maioria (72.1\%) tem companheiro, $69.4 \%$ reside em meio urbano, $76.1 \%$ reside no seu país de origem, $45.9 \%$ possui ensino superior, $37.4 \%$ tem o ensino secundário, $78.2 \%$ estão ativas profissionalmente e $56.3 \%$ pratica alguma religião.

Refere-se que a grande maioria (81.1\%) não tem dificuldade em descolocar-se ao Centro de Saúde para vigilância da gravidez. Metade das participantes que admitem ter dificuldade em se deslocar ao Centro de Saúde e justificam-no com facto de não poderem faltar ao trabalho. Constata-se que $96.4 \%$ não consome bebidas alcoólicas e 81.5\% não tem hábitos tabágicos. No que diz respeito à prática de exercício físico, 56.8\% são praticantes. Relativamente à alimentação $54.5 \%$ classifica-a como moderadamente saudável, destacando-se as que a classificam como saudável/muito saudável (36.5\%). No que diz respeito à forma como classificam a sua saúde, em geral, apura-se que estão em maioria (59.0\%) as que a classificam como boa, enquanto $28.8 \%$ a classificam como muito boa/ótima e 12.2\% razoável/má. A quase totalidade da amostra (84.2\%), refere que no último trimestre não teve nenhuma doença. Relativamente à toma de medicação, $72.1 \%$ refere não tomar. Verifica-se ainda que a maioria (66.7\%), não tem história de doença familiar.

Constata-se que a maioria (63.1\%) admite ser esta a primeira gravidez, referindo $64.6 \%$ não ter tido complicações na gravidez. Verifica-se também que $63.4 \%$ não têm história de abortamento. Uma percentagem de $89.0 \%$ refere não ter tido necessidade de mais 2 anos para engravidar. Quanto ao tipo de parto, 29.8\% tive um parto distócico e 40.2\% um parto eutócico. Relativamente à frequência de consultas de vigilância pré-concecio- 
nal, constatou-se que $55.0 \%$ as frequenta, enquanto $45.0 \%$ não frequentou, das quais $49.0 \%$ justifica com 0 facto de desconhecerem a importância dessas consultas; $14.0 \%$ refere que não frequenta as consultas de vigilância pré-concecional porque não as considera relevantes para si e para uma futura gravidez; $23.0 \%$ justifica com 0 facto de já ter conhecimento suficiente fruto de gravidezes anteriores; $27.0 \%$ considera não ter nenhum problema de saúde que possa interferir com a gravidez e, como tal, não frequentaram a consulta de vigilância pré-concecional. A maioria refere que a gravidez foi planeada/desejada, enquanto $31.5 \%$ admite que não. Constata-se ainda que a quase totalidade (92.8\%) não recorreu a tratamento de fertilidade. Mais de metade da amostra (77.0\%) não teve gravidez de risco. Verifica-se que a grande maioria (88.7\%), está a cumprir a vigilância de saúde pré natal recomendada, as que não o fazem apontam como motivo a falta de tempo, de conhecimento e desmotivação. Constata-se que $60.4 \%$ não frequentou curso para a parentalidade e parto.

A relação entre as variáveis sociodemográficas e a literacia em saúde mental positiva revela que as participantes mais velhas manifestam mais literacia em saúde mental positiva, mas sem diferenças estatisticamente significativas entre os grupos etários. Constatou-se que as participantes com companheiro revelam mais literacia em saúde mental positiva $(p=0,028)$. A zona de residência tem influência na literacia em saúde mental positiva, sendo as residentes em zona urbana as que apresentam índices superiores $(p=0,014)$. Os resultados relativos à influência das habilitações literárias na literacia em saúde mental positiva, revelam que as participantes com 0 ensino superior são as aquelas com melhores indicadores $(\mathrm{p}=0,000)$. Também as ativas profissionalmente revelam mais literacia em saúde mental positiva $(p=0,014)$. Constata-se que as que têm uma profissão intelectual manifestam mais literacia em saúde mental positiva, seguidas pelas que possuem uma profissão indiferenciada $(\mathrm{p}=0,017)$.

Na relação entre as variáveis contextuais à saúde e a literacia em saúde mental positiva, constata-se que as participantes que apresentam mais literacia em saúde mental positiva, não consomem bebidas alcoólicas, não têm dificuldade em se deslocar ao Centro de Saúde, avaliam a sua saúde como muito boa/ótima tiveram uma doença nos últimos 3 meses, tomam habitualmente medicação e têm história familiar de doença crónica, no entanto, sem relevância estatisticamente significativa. Pode-se afirmar que as participantes que não têm hábitos tabágicos e que classificam a sua alimentação como saudável/muito saudável revelam mais literacia em saúde mental positiva, com diferenças estatisticamente significativas.

Relativamente à relação entre as variáveis contextuais à gravidez e a literacia em saúde mental positiva verifica-se que as participantes multigestas, que necessitaram de mais de 2 anos para engravidar, que tiveram gravidez de risco, revelam mais literacia em saúde mental positiva, todavia, não existem diferenças estatisticamente significativas.

Por outro lado, as participantes que admitem que a sua gravidez foi planeada/desejada, que recorreram a tratamentos de fertilidade, com a gravidez vigiada pelo enfermeiro e o médico, que cumpriram a vigilância da gravidez recomendada, frequentaram um curso de preparação para a parentalidade e parto, apresentam mais literacia em saúde mental positiva, resultando em diferenças estatisticamente significativas.

\section{DISCUSSÃO}

A maioria das participantes (63.1\%) admitiu que esta é a sua primeira gravidez, $64.6 \%$ não teve complicações na gravidez. Constatou-se que 89.0\% não tiveram necessidade de mais 2 anos para engravidar. Na amostra, prevaleceu o parto eutócico (40.2\%). Constatou-se que prevalecem as participantes que frequentaram as consultas pré concecionais (55.0\%), de referir que $45.0 \%$ não as frequentou, das quais $49.0 \%$ justificam com o facto de desconhecerem a importância dessas consultas. Face a estes resultados importa referir que os profissionais de saúde, nomeadamente os enfermeiros, assumem um papel importante a este nível, informando as mulheres da importância da vigilância pré concecional, uma vez que a melhoria da qualidade dos cuidados prestados à grávida e ao recém-nascido é uma das áreas de intervenção prioritária do Plano Nacional de Saúde. Importa, desta forma, reforçar os cuidados dirigidos para o período pré concecional, uma vez que o ganho em saúde de uma intervenção sistemática e programada, nesta fase do ciclo de vida, é vital (Portugal, Ministério da Saúde, Direção- 


\section{CONHECIMENTOS DAS GRÁVIDAS EM SAÚDE MENTAL POSITIVA}

Geral da Saúde (2006). De acordo com Martins (2004), para além da prevenção de complicações, a consulta préconcecional proporciona ao casal toda a informação necessária para o período pré-concecional e gestação, o que faz aumentar a literacia em saúde. Também, planear a gravidez equivale à melhor maneira de prevenir riscos na gestação. A vigilância da gravidez deve iniciar-se precocemente para que os comportamentos de risco sejam alterados (Portugal, Ministério da Saúde, Direção-Geral da Saúde (2006).

Grande parte das participantes refere que a gravidez atual foi planeada/desejada (68.5\%), não tendo recorrido a tratamento de fertilidade (92.8\%). Constata-se que quase a totalidade da amostra não teve necessidade de mais de 2 anos para engravidar (92.3\%). Mais de metade da amostra (77.0\%) não teve gravidez de risco. Procurouse saber qual o profissional responsável pela vigilância da gravidez, verificando-se que, para a maioria, é o enfermeiro e o médico (69.8\%). Verificou-se que a grande maioria (88.7\%) está a cumprir a vigilância recomendada. De igual modo, no estudo de Silva, Bezerra, Rodrigues\& Araújo (2009), no que se refere à realização da vigilância da gravidez, ficou demonstrado que $84.3 \%$ das mulheres cumpriram a vigilância recomendada.

Constatou-se que $60.4 \%$ das mulheres não frequentam curso para a parentalidade e parto, contrariamente a 39.6\% que frequentam. Barge e Carvalho (2011) referem que a frequência de aulas de preparação para a parentalidade e parto aumentam o conhecimento e as competências das grávidas, possuindo maiores taxas de literacia em saúde. Acresce referir que, segundo Guerra et al. (2014), assume muita importância que as mulheres participem em cursos de preparação para a parentalidade em grupo, para que possam partilhar as suas experiências e aumentarem a sua rede de suporte social.

No que se refere à relação entre as variáveis sociodemográficas e a literacia em saúde mental positiva, constatou-se que as mulheres com companheiro, residentes em zona urbana, com o ensino superior, ativas profissionalmente e as que têm uma profissão intelectual revelam mais literacia em saúde mental positiva, com diferenças estatisticamente significativas.

Apurou-se que as participantes que não têm hábitos tabágicos e que classificam a sua alimentação como saudável/muito saudável revelam mais literacia em saúde mental positiva. Estes resultados poderão ser o reflexo do facto de possuírem literacia em saúde mental positiva, levando-as a estilos de vida mais saudáveis. A literacia em saúde mental possibilita aumentar o conhecimento e capacitar as pessoas para gerir de forma eficaz as condições de saúde ao longo da vida (Kutcher, Wei, \& Coniglio, 2016) Para Ferreira (2013), as grávidas com maior literacia em saúde são as que recorrem a mais fontes de informação, para esclarecerem dúvidas relativas à gravidez, parto e puerpério.

No que diz respeito à relação entre as variáveis contextuais à gravidez e a literacia em saúde mental positiva, constatou-se que existem diferenças estatisticamente significativas nas participantes que admitem que a sua gravidez foi planeada/desejada, que recorreram a tratamentos de fertilidade, as que 0 enfermeiro e 0 médico são quem vigia a sua gravidez, que cumprem a vigilância da gravidez recomendada e que frequentam um curso de preparação para a parentalidade e parto, sendo estas as que manifestaram mais literacia em saúde mental positiva. Estes resultados poderão ser justificados com o facto de que o acompanhamento durante a gravidez, por profissionais de saúde, assim como o terem frequentado um curso de preparação para a parentalidade e parto, Ihes permite adquirir conhecimentos que Ihes possibilita a compreensão e análise dos fatores inerentes ao processo gravídico, revelando comportamentos coincidentes com um bom desenvolvimento da sua gravidez e, como tal, reveladores de saúde mental positiva, que é um constructo que depende de quatro fatores relevantes, nomeadamente fatores e experiências individuais, interações sociais, estruturas e recursos da sociedade e valores culturais (Lahtinen, Lehtinen, Riikonen \& Ahonen, 1999).

\section{CONCLUSÕES}

0 período gravídico é um período de transição que requer a preparação para o desempenho de novos papeis e funções, implicando 0 assumir de novas responsabilidades. Tal facto, pode desencadear receios, inseguranças e inquietações, o que poderá implicar uma diminuição da saúde mental positiva da grávida, caso esta não tenha conhecimentos, habilidades e suporte profissional e social adequado. Estes resultados sugerem que os profis- 
sionais de saúde em geral, e os enfermeiros em particular, devem, no papel de promotores da saúde, intervir de forma sustentada na promoção de literacia em saúde mental positiva na mulher grávida, o que pode passar pela informação, orientação, aconselhando, esclarecimento de dúvidas à mulher grávida para que esta tenha uma vivência saudável do seu processo gravídico, atendendo-se ao facto de que a gravidez se constitui como um momento em que emergem alterações físicas, hormonais, psicológicas e sociais, alterações estas que parecem influenciar diretamente a saúde mental da grávida. Pelo exposto, os profissionais de saúde têm um papel crucial ao dotar as grávidas de conhecimentos e competências que Ihes permitam vivências, transformações e interações normais ao longo do processo gravídico, o que se irá repercutir no período puerperal.

\section{REFERÊNCIAS BIBLIOGRÁFICAS}

Alderdice, F., MacNeill, J., \& Lynn, F. (2013). A systematic review of systematic reviews of interventions to improve maternal mental health and well-being. Midwifery, 29(4), 389-399. Acedido em https://linkinghub.elsevier.com/retrieve/pii/S02666138(12)00088-5

Barge, D., \& Carvalho, M. (2011). Prevalência e fatores condicionantes do aleitamento materno: Estudo ALMAT. Revista Portuguesa de Clinica Geral, 27(6), 518-525. Acedido em https://www.rpmgf.pt/ojs/index.php/rpmgf/article/view/10900

Brito, I. (2009) A saúde mental na gravidez e primeira infância. Revista Portuguesa de Clínica Geral, 25(5), 600604. Acedido em https://www.rpmgf.pt/ojs/index.php/rpmgf/article/view/10678

Cabral, L. R, \& Florentim, R. J. S. (2015). Saúde mental dos enfermeiros nos cuidados de saúde primários. Millenium, 49(20), 195-216. Acedido em https://revistas.rcaap.pt/millenium/article/view/8085

Chaves, C., Sequeira, C., Duarte, J. (2018). Projeto de Doutoramento em Ciências de Enfermagem "Avaliação dos níveis de literacia em saúde mental positiva na comunidade" Linha 1: Estudos epidemiológicos em Literacia em Saúde Mental Investigação em SM+Literacia - NURSID - CINTESIS. Instituto de Ciências Biomédicas Abel Salazar da Universidade do Porto.

Conde, A., \& Figueiredo, B. (2005). Ansiedade na gravidez: Implicações para a saúde e desenvolvimento do bebé e mecanismos neurofisiológicos envolvidos. Acta Pediátrica Portuguesa, 36(1), 41-49. Acedido em http://repositorium.sdum.uminho.pt/handle/1822/4646

Ferreira, S. R. R. (2013). Literacia na gravidez: Utilização da internet como fonte de informação (Dissertação de mestrado, Escola Superior de Enfermagem de Coimbra). Acedido em https://repositorio.esenfc.pt/rc/

Guerra, M. J., Braga, M.C., Quelhas, I., \& Silva, R. (2014). Promoção da saúde mental na gravidez e no pós-parto. Revista Portuguesa de Enfermagem de Saúde Mental, Espec.(1), 117-124. Acedido em https://repositorio.ucp.pt/handle/10400.14/18436

Jorm, A. F. (2012). Mental health literacy: Empowering the community to take action for better mental health. American Psychologist, 67(3), 231-243. http://dx.doi.org/10.1037/a0025957.

Jorm, A. F. (2014). Mental health literacy: Promoting public action to reduce mental health problems. In L. M. J. Loureiro (coord.), Literacia em saúde mental: Capacitar as pessoas e as comunidades para agir (pp. 27-39). Coimbra: Unidade de Investigação em Ciências da saúde.

Kohan, S., Ghasemi, S., \& Dodangeh, M. (2007). Associations between maternal health literacy and prenatal care and pregnancy outcome. Iranian Journal of Nursing and Midwifery Research, 12(4), 146-152. Acedido em http://www.ijnmr.mui.ac.ir/index.php/ijnmr/article/viewFile/31/31

Kutcher, S., Wei, Y., \& Coniglio, C. (2016). Mental health literacy: Past, present, and future. Canadian Journal of Psychiatry, 61( 3), 154-158. Doi: 10.1177/0706743715616609.

Lahtinen, E., Lehtinen, V., Riikonen, E., \& Ahonen, J. (1999). Framework for promoting mental health in Europe. Helsinki: Stakes.

Loureiro, I. (2015). A literacia em saúde, as políticas e a participação do cidadão. Revista Portuguesa de Saúde Pública, 33(1), 1. Acedido em https://www.elsevier.es/en-revista-revista-portuguesa-saude-publica-323pdf-S0870902515000206 
Martins, F. (2004). Um olhar sobre saúde reprodutiva em Portugal: 0 passado, o presente, que futuro? In VIII Congresso Luso-Afro-Brasileiro de Ciências da Saúde, A questão social no novo milénio. Coimbra: Universidade de Coimbra. Acedido em www.ces.uc.pt/lab2004/pdfs/FatimaMartins.pdf

Meleis, A. I., Sawyer, L. M. I. E. 0., Messias, D. K. H., \& Schumacher, K. (2010). Experiencing transitions: Emergency middle-range theory. In: A. I. Meleis, Transitions theory: Middle range and situation specific theories in nursing and practice (pp. 52-63). New York: Springer Publishing Company.

Portugal, Ministério da Saúde, Direção-Geral da Saúde. (2006). Circular Normativa n²/DSMIA de 16/01/2006: Prestação de cuidados pré-concepcionais. Lisboa: DGS. Acedido em https://www.dgs.pt/directrizes-dadgs/normas-e-circulares-normativas/circular-normativa-n-2dsmia-de-16012006.aspx

Silva, R. M., Bezerra, E. D., Rodrigues, D. P., \& Araújo, M. A. L. (2009). Consulta pré-natal na perspectiva de gestantes em uma regional de saúde de Fortaleza-Ceará. Cadernos de Saúde Coletiva, 17(4), 1001-1015. Acedido em http://www.cadernos.iesc.ufrj.br/cadernos/images/csc/2009_4/artigos/Artigo_6.pdf 